\title{
Programa educativo PRIRES: Programa de Prevención de Riesgos en las Redes Sociales
}

PRIRES education program: Risk prevention program of social networks

\author{
Dr. José María Avilés Martínez ${ }^{1}$
}

Universidad de Valladolid

(RECIBIDO 04/04/2014, ACEPTADO 08/10/2014)

\begin{abstract}
RESUMEN
El artículo ofrece una reflexión sobre los procesos de carácter moral subyacentes a las relaciones interpersonales que se ponen en juego en la convivencia escolar en general y en las situaciones de violencia entre iguales (bullying y cyberbullying) en particular. Presenta el programa educativo PRIRES para trabajar esos procesos en la comunidad educativa.
\end{abstract}

Palabras clave: Bullying, convivencia escolar, cyberbullying, desarrollo moral, redes sociales, programas de prevención.

\begin{abstract}
The article offers a reflection on the underlying processes of moral character to the interpersonal relations that are brought into play in the school day-to-day life in general and in situations of peer violence (bullying and cyberbullying) in particular. It presents the educational program PRIRES so as to work these processes in the school community.
\end{abstract}

Keywords: Bullying, living together in school, cyberbullying, moral development, social networks, programs of prevention. 


\section{INTRODUCCIÓN Y JUSTIFICACIÓN}

La potencialidad de las redes sociales por una parte ha positivado las dimensiones comunicativas y de relaciones entre nuestros estudiantes, favoreciendo dimensiones como el conocimiento, la relación, el contagio que proporciona estar en red, el aprendizaje, la disposición de la información, la amistad o la influencia.

Sin embargo, por otra parte, en ellas se han visualizado diferentes riesgos que su uso indiscriminado y sin control o con ausencia de sentido común, han hecho patentes. Hablamos de dependencia, adicciones, hiperconexión, hipervirtualidad, distancia emocional, inmediatez-impulsividad, desconexión moral, conducta dual o cyberbullying. Éste último es uno de los más preocupantes y que afecta a nuestros escolares.

El traslado de la convivencia entre escolares a los espacios que brindan las redes sociales a través de diferentes dispositivos móviles, por una parte ha ubicado en ese escenario problemas antiguos como el bullying, que tradicionalmente venían dándose en las relaciones interpersonales presenciales entre escolares, y por otra, ha aportado nuevas dimensiones psicosociales al maltrato entre iguales en los planos interpersonal, intrapersonal, grupal y contextual (Avilés, 2013b; Mason, 2005) que han modulado las conductas, emociones, actitudes, intervenciones y posicionamientos morales de los involucrados que participan en él. Estas formas acoso a través de la red ha adoptado diversas formas (Willard, 2006), hablamos de hostigamiento (envío y difusión de mensajes ofensivos o vulgares) persecución (envío de mensajes amenazantes), denigración (difusión de rumores), violación de la intimidad (difusión de secretos o imágenes), exclusión social (exclusión deliberada de grupos en la red), suplantación de la identidad (envío de mensajes maliciosos haciéndose pasar por otro), etc.

La irrupción del ciberacoso en los contextos escolares y sus entornos está obligando a los agentes educativos a situar de forma adecuada el problema del maltrato entre iguales, sea presencial o virtual, y que no es otro que frente a sus dimensiones morales y a la toma de decisiones implícitas o explícitas de todos y cada uno de quienes conocen de él, sean testigos, victimizados o victimarios. Algunas comunidades educativas se han posicionado frente al cyberbullying en esta perspectiva, considerándolo como un problema de convivencia escolar con dimensiones evidentemente morales, independientemente de dónde esté su origen. Así han ido legitimando a los agentes educativos comprometiéndolos para intervenir de forma participativa y responsable en los procesos correctores desde perspectivas, en la medida de lo posible restauradoras de las relaciones. Otras no han abordado el problema educativo y colectivamente o no han sabido cómo, aunque han tenido que darle respuesta conforme lo han ido enfrentando para resolver situaciones que protagonizaba su propio alumnado. También existen comunidades que lo niegan u ocultan o sitúan su competencia lejos de la intervención educativa aduciendo que muchas de las acciones se producen o provienen de fuera del entorno estrictamente escolar. Esta ausencia de intervenciones educativas ha trasladado a otros árbitros la resolución de los casos y ha favorecido una perspectiva más punitiva, legalista o, incluso policial de los mismos. 
Desde nuestro punto de vista se hace necesario trabajar en la escuela de forma preventiva procesos psicosociales subyacentes a las situaciones de riesgo que ocurren en las redes sociales, incluido del cyberbullying, cuando los adolescentes han acabado situando en ellas gran parte de sus interacciones interpersonales. Entendemos que este trabajo deben hacerlo los agentes educativos coordinadamente de forma planificada e inserto en el currículo escolar, y tiene que incidir en los procesos de toma de decisiones morales de los adolecentes (Avilés, Irurtia, $\mathrm{G}^{\mathbf{a}}$ López y Caballo, 2011) cuando estos gestionen sus relaciones interpersonales en los entornos virtuales. En esta línea y con esa intención de incidir en la conducta moral, otros programas son pioneros en abordar la prevención e intervención de problemas de conducta o conductas de riesgo como bullying, violencia, conductas antisociales, etc. (DiBiase, Gibbs y Potter, 2010; Segura, Arcas y Mesa, 1998; Segura, 2007). Nuestro interés es centrar ese trabajo en los contextos virtuales de las redes sociales y especialmente en el cyberbullying como manifestación del abuso en ellas. Venimos haciéndolo desde los instrumentos de planificación pedagógica de los centros educativos y desde los foros formativos de las comunidades educativas en torno a un programa educativo de prevención de riesgos en las redes sociales -Prires- (Avilés, 2013a), a partir de sesiones de trabajo con el alumnado, el profesorado y las familias, que persigue ayudarles a construir herramientas de gestión de las situaciones que habitualmente enfrentan en sus interacciones en las redes sociales, desde una perspectiva de desarrollo moral.

Esta comunicación pretende esbozar los elementos nucleares de este programa haciendo hincapié fundamentalmente en el trabajo que el profesorado realiza con el alumnado.

\section{OBJETIVOS}

Pretendemos poner de relevancia la importancia de considerar los aspectos morales en los programas preventivos y de intervención con el alumnado referidos a las conductas de riesgos de los adolecentes en las redes sociales. Hacerlo de forma intencional y como parte del currículo del alumnado es lo que intentamos esbozar a través de los contenidos de trabajo básicos del programa educativo Prires (Prevención de riesgos en las redes sociales).

\section{MARCO TEÓRICO - ANTECEDENTES}

La intervención en educación moral como objetivo educativo a través de programas formalizados para transformar situaciones de riesgo, conflicto, violencia o conductas antisociales en realidades personales y sociales más sanas y moralmente aceptables, se ha inspirado en planteamientos teóricos diversos y complementarios (Escámez, 2003). Son tradiciones filosóficas y científicas respetadas en el ámbito del conocimiento y han puesto el acento preferentemente en diferentes focos de actuación, concediendo más peso en la intervención a los procesos individuales que comunitarios o más al trabajo sobre los contenidos cognitivos que socioemocionales. 
Así, desde una perspectiva más clásica, se aporta una concepción más comunitaria en que la relación entre el individuo y la comunidad en que vive se plantea como recíproca en las influencias y son las acciones de los individuos que se plasman en la convivencia lo que sería el objeto de intervención en la escuela. Las relaciones que los individuos establecen con sus grupos de referencia y cómo estos comparten y construyen prácticas, costumbres y valores. Las redes sociales funcionarían desde esta perspectiva respecto a los individuos tomados de forma singular (Aguilar y Said, 2010). Les proporcionan la identidad social virtual y estos se sienten acogidos y reconocidos en ella. Desde esta perspectiva, la intervención en educación moral en la escuela aprovecha todas las iniciativas que ayudan a vincular al individuo con la comunidad y la identidad social que esta reconoce en los individuos, y pone a su servicio algunos instrumentos sociales, pedagógicos y psicológicos que ayudan a conseguirlo, como el trabajo cooperativo, las normas, los sentimientos de pertenencia al grupo, el clima de aula, la responsabilidad social, la participación y clima democrático, etc.

Otra perspectiva de trabajo de la educación moral en la escuela pone el énfasis como señala Escámez (2003) en los aspectos de progreso individual en la construcción de su desarrollo moral (Kolhberg y Kramer, 1969). El trabajo de la escuela acompañaría al individuo, aunque sea en contextos sociales, en ese proceso para avanzar hacia la justicia y los principios universales. Kolhberg, propone ese avance estructura a estructura y construcción a construcción desde estadíos preconvencionales hasta los postconvencionales. Desde el punto de vista escolar esta perspectiva entiende que la escuela debe brindar a los estudiantes oportunidades de reflexión que les permitan avanzar en la autonomía moral resolviendo los conflictos de convivencia a partir del debate y resolución colaborativa con otros, pero asegurando el avance individual.

Un enfoque más reciente fue el conocido como Filosofía para niños (Lipman y Sharp, 1992) que pretende aportar herramientas para aprender a pensar con significado y sentido crítico, para que el alumnado construya respuestas en su realidad individual y social. Desde esta perspectiva el énfasis sobre la educación moral en la escuela recae en proporcionar materiales sesiones o situaciones en las que el alumnado pueda construir respuestas moralmente aceptables a situaciones extraídas de su vida cotidiana a partir de cuestionamientos. Este enfoque aportaría al trabajo preventivo sobre los riesgos en las redes sociales fundamentalmente la toma de conciencia de los problemas morales a los que se enfrentan habitualmente cuando toman decisiones individuales y sociales, por acción o por omisión, y una necesidad de mejorar las habilidades para la gestión de la convivencia con las que comprometan a dar soluciones justas y equilibradas.

Otras perspectivas han incorporado más recientemente al trabajo de educación moral a partir de programas estructurados, ámbitos tan relevantes como el de las emociones y su gestión en la convivencia escolar en general (Bisquerra, 2008; Extremera y Fernández-Berrocal, 2003) y el del maltrato entre iguales en particular (Bisquerra, Colau, Colau, Collell, Escudé, Pérez-Escoda, Avilés y Ortega, 2014). También han sido importantes las aportaciones que desde la competencia 
social y el entrenamiento en habilidades sociales (Goldstein, Sprafkin, Gershaw y Klein, 1989; Segura, 2007) se han hecho para la integración en el currículo de la escuela de programas estructurados que abordan el desarrollo moral del alumnado intencionalmente. Y es desde la competencia social desde donde tenemos intención de aportar a la gestión de la convivencia en las escuelas, herramientas de trabajo con el alumnado que aborden los problemas de la ciberconvivencia a través de las redes sociales y del maltrato entre iguales en el ámbito virtual (cyberbullying), desde una perspectiva moral. Estas herramientas tocan los procesos que subyacen a esas situaciones a partir del tratamiento de contenidos en una serie de sesiones escolares, estructuradas en un programa -Prires- (Avilés, 2013a), e integradas en el currículo escolar de los centros.

\section{PROPUESTA EDUCATIVA - EL PROGRAMA}

El programa educativo Prires (Prevención de Riesgos en Redes Sociales) es un proyecto a aplicar en los centros escolares y dirigido al trabajo con estudiantes que inician y desarrollan sus relaciones interpersonales a través de los dispositivos móviles en las redes sociales.

El programa lo aplica el profesorado de los centros aunque también contempla la participación de alumnado (cybermentores) y familias para llevar a cabo y/o colaborar en la realización de algunas de sus tareas o sesiones (Avilés, 2014). Los centros que ponen en práctica el programa han optado por asumir su responsabilidad de gestión e intervención sobre los casos y las consecuencias que afecten a la convivencia del alumnado, aunque procedan de espacios privados y/o ajenos al centro escolar, derivadas de la gestión de las redes sociales y de las dinámicas negativas que puedan surgir de ellas. Del mismo modo, las comunidades educativas se han posicionado públicamente contra el acoso y el cyberbullying como formas de imposición y dominio-sumisión a través de declaraciones públicas y compromisos educativos explícitos en sus proyectos educativos. Finalmente, las comunidades educativas han tomado decisiones integradoras sobre el uso de los dispositivos móviles en la convivencia cotidiana y el desarrollo del currículo del centro.

\section{Orientación de la aplicación}

Preferentemente la orientación de la intervención educativa a través del programa es preventiva. Quienes lo aplican conocen y habitualmente conviven con el alumnado. El programa discurre integrado en el Plan de Acción Tutorial (PAT) del centro, sugerido y coordinado por el responsable del Grupo de Convivencia, Coordinador de Convivencia u Orientador/a del Centro. La orientación psicopedagógica del programa es restauradora (Avilés, 2012) y no culpabilizadora, por lo tanto, no punitiva y dirigida a brindar oportunidades al alumnado individualmente y en grupo para reflexionar y tomar decisiones de forma crítica y comprometida sobre sus prácticas cotidianas en las redes sociales y/o frente a situaciones de cyberbullying en las que son espectadores o protagonistas. 


\section{Destinatarios}

El alumnado destinatario del programa es tanto de Educación Primaria como de Educación Secundaria que gestiona y desarrolla sus relaciones interpersonales a través de las redes sociales. El momento para comenzar a aplicar el programa en el aula es el del inicio de los estudiantes en el uso de las redes sociales. El carácter de la aplicación debe ser crítico, reflexivo y preventivo sobre algunos de los riesgos a los que se van a enfrentar y sobre situaciones prácticas concretas en las que pueden verse envueltos.

\section{Líneas de actuación del programa}

- Carácter preventivo de la intervención: el programa busca anticiparse a las situaciones de riesgo a las que el alumnado puede enfrentarse en la gestión de sus relaciones en las redes sociales.

- Criterio moral: la educación moral desde el punto de vista formativo en la toma de decisiones autónoma de los estudiantes, es el fin último del programa, más allá de las situaciones o casos que se valoren o enfrenten.

- Efecto sumativo y formativo: el itinerario formativo que el alumnado realiza con la aplicación del programa viene reforzado de las intervenciones de todos los agentes educativos (profesorado y familias) en una misma dirección (lealtad educativa) y propiciado por la formación sobre los procesos a los que deben enfrentarse los estudiantes.

- Acompañamiento y supervisión: son tareas de compromiso educativo de profesorado y familias en el proceso de iniciación y presencia de los estudiantes en la gestión de sus relaciones en las redes sociales.

- Educación digital: se persigue proporcionar oportunidades para enriquecer el crecimiento digital del alumnado (imagen digital, identidad social, prácticas saludables, códigos virtuales, comunicación virtual, ...) desde la educación en valores.

- Contenidos de trabajo: abordaje psicopedagógico de un catálogo de temas relevantes en los procesos subyacentes a las situaciones de riesgos en las redes sociales y el cyberbullying.

\section{Procedimiento}

Las sesiones de aplicación del programa se realizan generalmente a partir de la metodología del análisis de casos (Avilés, 2012) o de valoración de situaciones concretas para el alumnado. Se presenta un caso a través de un soporte, frecuentemente, un video , un texto, un relato, una canción, ... y se propone primero un trabajo individual de reflexión, elaboración, crítica o valoración. Posteriormente se facilita un debate en pequeño grupo y finalmente se contrastan en gran grupo las ideas relevantes llegando a reconocimiento de aprendizajes y adopción de compromisos individuales y colectivos. La dramatización y el role play son herramientas habituales. 
La aplicación del programa se realiza en un número variable de sesiones dentro del currículo del alumnado en las que se trabajan los contenidos que lo conforman. El perfil del profesorado que lo lidera está relacionado con la gestión de la convivencia y previamente ha sido capacitado y entrenado en el manejo de los temas a tratar. Simultáneamente participa alumnado preparado previamente (cybermentores) al que se ha formado, y tienen experiencia en redes sociales. Es alumnado que en momentos anteriores ha integrado otros sistemas de apoyo en la escuela (Avilés, Torres y Vián, 2008) como Equipos de Ayuda, Mediación, etc. y que realiza la formación entre iguales y la tutorización de alumnado de menor edad, aportando su guía y experiencia conjuntamente con el profesorado en momentos concretos de la aplicación del programa. En paralelo, se realizan seminarios para familias abordando los mismos contenidos y aportando pautas de acompañamiento y supervisión para el trabajo educativo en casa.

Las sesiones de trabajo se realizan durante la tutoría o en sesiones específicas, estando presentes en ellas el profesorado tutor/a, el alumnado cybermentor y el profesor/a que conduce la sesión y que ha coordinado previamente la actuación.

\section{Contenidos de trabajo}

Habitualmente cuando hablamos de redes sociales e incluso de cyberbullying desde el punto de vista de la prevención el discurso se suele limitar al ámbito de los riesgos a los que los estudiantes pueden enfrentarse en las redes sociales, Sin embargo, nuestra mirada formativa va dirigida a proporcionar criterio y herramientas al alumnado para que pueda tomar decisiones correctas ante esas situaciones y eso pasa por el abordaje de determinados contenidos desde una perspectiva moral que son centrales en el Programa.

En cada módulo formativo se trabajan variables que luego son indicadores de evaluación.

\section{Privacidad}

El programa plantea el tratamiento de la privacidad abordándola como derecho y deber a partir de la imagen e identidad digitales. Trabaja el concepto de espacio público - espacio privado, la posesión de los contenidos en las redes sociales y las posibilidades de modificación y rectificación (capacidad de control y perdurabilidad), los contenidos de la información (contenidos sensibles) o las des-conexiones morales propias y ajenas a las que se enfrentan quienes conviven en las redes sociales.

\section{Comunicación}

El trabajo respecto a los procesos comunicativos se centra en que el alumnado aprenda a establecer y diferenciar los círculos de confianza, adoptando pautas de comunicación, códigos de práctica comunicativa y garantías de seguridad técnica y emocional. 


\section{Empatía virtual}

El trabajo para poner al alumnado en perspectiva emocional se encauza en el programa, ayudándoles a partir de situaciones concretas y a reconocer virtualmente signos emocionales, usar códigos emocionales activando elementos de comunicación virtual y la gestión virtual de las emociones (emoticonos, voz, etc.).

\section{Pensamiento consecuencial}

El trabajo en este ámbito se enfoca a que el alumnado adopte criterios morales para valorar el alcance de sus acciones. Es fundamental el entrenamiento en la anticipación de consecuencias, control de la impulsividad, adopción de respuestas equilibradas y ejercicio del pensamiento medios-fines.

\section{Autorregulación}

Este campo se trabaja en el programa con sesiones de autoconocimiento y autoaceptación a través de la metodología de preguntas y respuestas. El programa prevé sesiones de control de las emociones y trabajo de la resiliencia así como la ejercitación del uso de acuerdos a través de los seguimientos tutoriales y familiares y los acuerdos reeducativos (Alonso y Avilés, 2012). El tratamiento de la autosuficiencia tecnológica es otra premisa del trabajo que se realiza en este ámbito de contenidos.

\section{Riesgos de internet}

Quizás sea este abanico de temas de los más divulgados y conocidos en el tratamiento que los riesgos de las redes sociales que se tienen en muchos centros educativos. Sin embargo, en nuestro programa se consideran necesarios pero subsidiarios al tratamiento de los anteriores. Así, y en relación con los procesos subyacentes anteriores, también se abordan riesgos o prevenciones como la adopción de códigos online seguros, el afrontamiento de contenidos peligrosos en la red, la identificación de bulos (hoax), el tratamiento de virales, el pishing, la caracterización de las aplicaciones maliciosas, los efectos de las redes zombi, la identificación de publicidad engañosa (SMS Premium) y más pormenorizadamente el tratamiento y la prevención de situaciones extremas y peligrosas como el sexting, grooming, cyberbulying, happy slapping, etc.

\section{APLICACIÓN Y RESULTADOS}

Los datos que referimos a continuación pertenecen al pilotaje del programa, llevado a cabo en un centro de secundaria, en sus cuatro niveles, a través de un equipo tutores/as previamente entrenados (grupo de convivencia) y que realizan esas funciones tutoriales de convivencia con el alumnado. En el centro funcionan de manera normalizada sistemas de Equipos de Ayuda, Mediación y Cybermentores 
(Avilés, 2014). El alumnado participante asciende a 418, distribuido en $1^{\circ}$ (99 $-23,68 \%-), 2^{\circ}(109-26,07 \%-), 3^{\circ}$ (94 -22,48\%) y $4^{\circ}(116-27,75 \%)$ de la ESO, siendo el $48,80 \%$ de ellos chicos (204) y el 51,2\% chicas (214).

Los tiempos de aplicación en el centro piloto han sido ocho sesiones, en horas de tutoría de los grupos, durante un curso escolar. Antes y después de la aplicación del programa el alumnado ha completado en la intranet del centro un autoinforme que explora hábitos, posicionamientos y prácticas del alumnado en las redes sociales. El tiempo transcurrido entre el pretest y el postest ha sido de cinco meses, en que se desarrolla el programa. Tras el tratamiento estadístico de los datos a través del programa SPSS 20.0, los resultados que exponemos aquí son generales e iniciales, estando pendientes de desarrollarse más adelante en otros informes. De los datos, observamos que:

- El alumnado participante en el programa muestra tras su realización una mayor concienciación sobre los riesgos en las redes sociales y en internet y sobre su capacidad para tomar decisiones que afectan a uno mismo y a otros.

- Quienes realizan el programa se declaran con más recursos para afrontar una situación que les afecte a ellos en internet.

- En el ámbito de la intimidad, encontramos diferencias significativas ( $p<0.005)$. Tras haber aplicado el programa se aprecia que el alumnado reconoce con más facilidad situaciones donde están en juego conceptos como 'espacio público-espacio privado', y 'contenidos sensibles a comunicar por internet'.

- La puesta en marcha y aplicación del programa ha rebajado significativamente el número de incidentes entre el alumnado, estos están registrados en la aplicación informática de la Administración sobre la convivencia escolar en el centro, todos relacionados con conflictos referidos a las relaciones interpersonales del alumnado a través de las redes sociales.

- En cuanto a comunicación, encontramos diferencias significativas ( $p<0.001$ ) respecto a la extensión de los círculos de confianza en la comunicación de información personal, resultando más restrictiva después de la aplicación del programa.

- Se observan diferencias $(\mathrm{p}<0.005)$ después de la aplicación del programa en cuanto a la conciencia que el alumnado tiene de las consecuencias de sus acciones sobre cómo pueden sentirse los demás, lo que incide en su conciencia moral y empatía. Reconocen que piensan más en el/la otro/a antes de realizar una acción (enviar un mensaje, mandar un video, etc.).

- No encontramos diferencias significativas ni en el reconocimiento ni en el uso de códigos emocionales virtuales después de la aplicación del programa.

- Encontramos mejores puntuaciones respecto a los efectos de la aplicación del programa en cursos inferiores (primer ciclo de la ESO) que en superiores $\left(3^{\circ}\right.$ y $4^{\circ}$ de la ESO), lo que nos orienta a los momentos preferentes de aplicación. 


\section{CONCLUSIONES}

Dada la poca vigencia del programa en el centro, consideramos que tenemos que tomar con prudencia los resultados expuestos, ya que nos anima a apoyar su continuidad por la estabilidad en el tiempo de los programas de prevención y lucha contra el bullying para garantizar mejor la visibilidad de sus efectos (Smith, Pepler y Rigby, 2004). Igualmente será necesario evaluar su aplicación y efectos en otros centros y muestras más amplias, diversas y representativas.

Por otra parte, su aplicación refuerza la implementación de algunas líneas de trabajo en la prevención de situaciones de riesgo en las redes sociales en adolescentes. Encontramos que deben ser los agentes educativos (familias y profesorado) quienes coordinadamente asuman la intervención para señalar el trabajo preventivo respecto a pautas educativas y de compromiso en la misma dirección (Avilés, 2013c). Esto es especialmente importante cuando el alumnado se tiene que posicionar en las redes sociales ante casos de bullying entre colegas conocidos o cercanos (Olweus y Limber, 1999). Ello refuerza el trabajo de acompañamiento de los agentes educativos sobre el desarrollo moral de los sujetos como objetivo de la intervención. Ese trabajo debe estar preferentemente inserto en el currículo, agenda y planes educativos del centro, participando en él el mayor número posible de miembros, y además en los espacios y tiempos educativos de las familias. Esta tarea debe orientarse a acompañar la toma de decisiones morales del alumnado en sus relaciones interpersonales en línea y que en el contexto escolar afectar a la totalidad del grupo de convivencia como núcleo formativo, en su búsqueda adoptar compromisos individuales y colectivos verificables.

\section{REFERENCIAS BIBLIOGRÁFICAS}

Aguilar, D.E., Said, E. (2010). Identidad y subjetividad en las redes sociales virtuales: caso de Facebook En :<http://www.redalyc.org/articulo.oa?id=85316155013> (Recuperado el 9 de febrero de 2014)

Alonso, F. y Avilés, J. M a (2012). "Los acuerdos reeducativos como instrumento preferente de resolución de las situaciones de indisciplina escolar". En J.L. Pais, I. Leal, A. Pereira, A. Torres, I. Direito y P. Vagos (Eds.) $9^{\circ}$ Congreso de Psicología da Saude, pp. 400-406, Aveiro: Placebo.

Avilés, J. Ma (2012). Manual contra el bullying. Guía para el profesorado. Lima: Libro Amigo.

Avilés, J.M ${ }^{\mathrm{a}}$, (2013a, agosto). Educação para a convivência. Ponencia presentada en el Seminario Internacional 'A convivência na escola em pauta', Campinas, SP.

Avilés, J.Ma , (2013b). Análisis psicosocial del cyberbullying. Claves para una educación moral. Papeles del psicólogo, 34 (1), 65-73.

Avilés, J.M a , (2013c, abril 1). Bullying y cyberbullying: apuntes para la elaboración de un Proyecto Antibullying. CONVIVES, 3 (1), 4-15. Extraído el 16 de enero de 2014 desde http://convivenciaenlaescuela.es/?page_id $=541$ 
Avilés, J.M a (2014, enero). Redes Sociales y Ciberconvivencia. Ponencia presentada en la I Jornada Provincial de Convivencia en los Centros, Valladolid.

Avilés, J. M., Torres, N. y Vian, M.V. (2008). Equipos de ayuda, maltrato entre iguales y convivencia escolar. Electronic Journal of Reasearch in Educational Psychology, 6, (3), 357-376.

Avilés, J.M ${ }^{\mathrm{a}}$, Irurtia, M.J., G ${ }^{\mathrm{a}}$-López, J. y Caballo, V. (2011). Bullying, el maltrato entre iguales [Bullying], Behavioral Psychology, 19, (1), 57-90.

Bisquerra, R. (2008). Educación para la ciudadanía y convivencia. El enfoque de la educación emocional. Barcelona: Wolters Kluwer.

Bisquerra, R. (coord.), Colau, C., Colau, P., Collell, J., Escudé, C., Pérez-Escoda, N., Avilés, J. M., y Ortega, R. (2014). Prevención del acoso escolar con educación emocional. Bilbao: Desclée de Brower.

Escámez, J. (2003). Pensar y hacer hoy educación moral. Teoría de la Educación. 15 21-31.

Extremera, N. y Fernández-Berrocal, P. (2003), la inteligencia emocional en el contexto educativo: hallazgos científicos de sus efectos en el aula. Revista de Educación, 332, 97-116.

Goldstein, A., Sprafkin, R., Gershaw, N., Klein, P. (1989). Habilidades sociales y autocontrol en la adolescencia. Un programa de enseñanza. Barcelona: Martínez Roca.

Kohlberg, L. y Kramer, R. (1969). Continuities and discontinuities in childhood and adult moral development, Human Development, 12, 93-120. doi: 10.1159/000270857

Lipman y Sharp (1992). La filosofía en el Aula. Madrid: Editorial de la Torre.

Mason, T. (2005). How to teach children at-risk of educational failure: Coping with poverty, bullying, disease, crime and ethnicity. Lewiston, NY: The Edwin Mellen Press.

Olweus, D., y Limber, S. (1999). Blueprints for violence prevention: Bullying Prevention Program. Boulder, CO: Institute of Behavioral Science.

Segura Morales, M., Arcas Cuenca, M. y Mesa Expósito, J.R. (1998.) Programa de competencia social. Habilidades cognitivas, valores morales y habilidades sociales. Educación Secundaria. Materiales. Canarias: Consejería de Educación, Cultura y Deportes del Gobierno de Canarias.

Segura Morales, M. (2007). Jóvenes y adultos con problemas de conducta. Desarrollo de las competencias sociales. Madrid: Narcea ediciones.

Smith, P.K., Pepler, D. y Rigby, K. (2004). Bullying in schools. Cambridge: University Press. 